\title{
Reduced parahippocampal cortical thickness in subjects at ultra-high risk for psychosis
}

\author{
S. Tognin ${ }^{1 *}$, A. Riecher-Rössler ${ }^{2}$, E. M. Meisenzahl ${ }^{3}$, S. J. Wood ${ }^{4,5}$, C. Hutton ${ }^{6}$, S. J. Borgwardt ${ }^{2}$, \\ N. Koutsouleris ${ }^{3}$, A. R. Yung ${ }^{7}$, P. Allen ${ }^{1}$, L. J. Phillips ${ }^{8}$, P. D. McGorry ${ }^{7}$, I. Valli ${ }^{1}$, D. Velakoulis ${ }^{4}$, \\ B. Nelson ${ }^{7}$, J. Woolley ${ }^{1}$, C. Pantelis ${ }^{4}$, P. McGuire ${ }^{1}+$ and A. Mechelli ${ }^{1}+$ \\ ${ }^{1}$ Department of Psychosis Studies, Institute of Psychiatry, King's College London, London, UK \\ ${ }^{2}$ Center for Gender Research and Early Detection, University of Basel Psychiatric Clinics, clo University Hospital Basel, Petersgraben, Basel, \\ Switzerland \\ ${ }^{3}$ Departments of Psychiatry and Psychotherapy, Ludwig-Maximilians-University, Munich, Germany \\ ${ }^{4}$ Melbourne Neuropsychiatry Centre, Department of Psychiatry, The University of Melbourne and Melbourne Health, Victoria, Australia \\ ${ }^{5}$ School of Psychology, University of Birmingham, Birmingham, UK \\ ${ }^{6}$ Wellcome Trust Centre for Neuroimaging, UCL Institute of Neurology, University College London, London, UK \\ ${ }^{7}$ Orygen Research Centre, University of Melbourne, Victoria, Australia \\ ${ }^{8}$ Psychological Sciences, University of Melbourne, Victoria, Australia
}

Background. Grey matter volume and cortical thickness represent two complementary aspects of brain structure. Several studies have described reductions in grey matter volume in people at ultra-high risk (UHR) of psychosis; however, little is known about cortical thickness in this group. The aim of the present study was to investigate cortical thickness alterations in UHR subjects and compare individuals who subsequently did and did not develop psychosis.

Method. We examined magnetic resonance imaging data collected at four different scanning sites. The UHR subjects were followed up for at least 2 years. Subsequent to scanning, 50 UHR subjects developed psychosis and 117 did not. Cortical thickness was examined in regions previously identified as sites of neuroanatomical alterations in UHR subjects, using voxel-based cortical thickness.

Results. At baseline UHR subjects, compared with controls, showed reduced cortical thickness in the right parahippocampal gyrus ( $p<0.05$, familywise error corrected). There were no significant differences in cortical thickness between the UHR subjects who later developed psychosis and those who did not.

Conclusions. These data suggest that UHR symptomatology is characterized by alterations in the thickness of the medial temporal cortex. We did not find evidence that the later progression to psychosis was linked to additional alterations in cortical thickness, although we cannot exclude the possibility that the study lacked sufficient power to detect such differences.

Received 13 September 2012; Revised 6 April 2013; Accepted 11 April 2013; First published online 10 May 2013

Key words: Cerebral cortex, magnetic resonance imaging, parahippocampal gyrus, prodromal period, schizophrenic psychoses, voxel-based cortical thickness.

\section{Introduction}

The first episode of a psychotic disorder is usually preceded by a prodromal period characterized by a progressive decline in functioning and the emergence of attenuated psychotic symptoms. Individuals with these clinical features are said to be at 'ultra-high risk' (UHR) for psychosis because $18-36 \%$ of them will develop a psychotic disorder within 3 years

* Address for correspondence: S. Tognin, Department of Psychosis Studies, PO Box 67, Institute of Psychiatry, King's College London, De Crespigny Park, London SE5 8AF, UK.

(Email: stefania.tognin@kcl.ac.uk)

+ These authors contributed equally to this work.
(Fusar-Poli et al. 2012). Early clinical intervention in the UHR population may reduce the risk of later transition to psychosis and improve long-term clinical and functional outcome (McGorry et al. 2009); however, at present it is difficult to predict based on first clinical presentation which UHR individual who will and will not go on to develop psychosis. Recent work has sought to examine if neuroimaging can be used to identify individuals at UHR and predict who will subsequently make transition to psychosis within this population (Koutsouleris et al. 2009).

Studies using voxel-based morphometry (VBM) have examined whether UHR subjects are affected by neuroanatomical abnormalities. Cross-sectional studies indicate that, relative to healthy controls, UHR subjects 
have reduced grey matter (GM) volume in frontal (Meisenzahl et al. 2008; Mechelli et al. 2011), lateral and medial temporal regions (Meisenzahl et al. 2008). Studies that used a region of interest (ROI) approach reported GM volume increases (Buehlmann et al. 2010) but also reductions (Phillips et al. 2002) in the hippocampus, reductions in the planum polare/ temporale, insula and superior temporal gyrus (Takahashi et al. 2009, 2010), increases in the pituitary volume (Büschlen et al. 2011), and reductions in the anterior cingulate cortex (ACC) (Röthlisberger et al. 2012) in the UHR group compared with healthy controls.

In VBM studies, relative to UHR subjects who did not develop psychosis (UHR-NT), those who later became psychotic (UHR-T) had reduced volumes in the inferior frontal cortex, medial and lateral temporal cortex, ACC (Pantelis et al. 2003), insular, inferior and superior frontal cortex (Borgwardt et al. 2008). ROI studies suggest that later transition to psychosis is associated with reduced volume in the left parahippocampal gyrus (Mechelli et al. 2011), the insula bilaterally (Takahashi et al. 2009), the left ACC (Röthlisberger et al. 2012), and with increased pituitary (Büschlen et al. 2011) and hippocampus (Buehlmann et al. 2010) volumes.

Some ROI studies did not find any significant differences between UHR subjects and healthy controls (Wood et al. 2005; Velakoulis et al. 2006) or between UHR-T and UHR-NT individuals (Yucel et al. 2003; Velakoulis et al. 2006). Nevertheless, evidence that there may be volumetric differences between the latter subgroups raises the possibility that neuroimaging measures might be able to facilitate the prediction of clinical outcome in UHR subjects.

The vast majority of structural neuroimaging studies in UHR individuals have focused on GM volume. However, neuroanatomical alterations in psychosis may be expressed not only in terms of GM volume but also as subtle changes in cortical thickness. While the analysis of GM volume returns a mixed measure that depends on local cortical thickness as well as cortical folding and gyrification (i.e. cortical surface area), the analysis of cortical thickness is considered to specifically target the presence of cortical atrophy (Hutton et al. 2009). Therefore the two approaches provide complementary information and one can be more sensitive than the other depending on the process underlying neuroanatomical changes. Cortical thickness in the human brain can be examined using the automated data analysis of $\mathrm{T}_{1}$-weighted images (Hutton et al. 2008). Application of this approach in patients with first-episode and established schizophrenia suggests that there is a cortical thinning in the anterior cingulate (Narr et al. 2005a; Fornito et al. 2008a; Schultz et al. 2010b), prefrontal (Narr et al. 2005a; Venkatasubramanian et al. 2008; Schultz et al. 2010b), temporal (Narr et al. 2005a; Fornito et al. 2008a) and occipital (Narr et al. 2005b) cortices. In UHR subjects, reduced cortical thickness has been reported in the prefrontal, ACC, inferior parietal, superior temporal and parahippocampal cortices compared with healthy controls (Jung et al. 2011). One study has reported that in UHR individuals who subsequently developed psychosis the ACC was thinner than in UHR-NT (Fornito et al. 2008b) and one has reported a progressive thinning in the anterior cingulate, precuneus and temporo-parietal-occipital cortex compared with UHR-NT and healthy controls (Ziermans et al. 2012). Another study did not find differences in cortical thickness between individuals at UHR, patients with a first psychotic episode and healthy controls, but the groups differed in cortical thickness asymmetry (Haller et al. 2009).

The findings from studies of cortical thickness in UHR subjects have thus been inconclusive, which may reflect the relatively small sample sizes examined to date. UHR subjects are difficult to recruit and it is therefore a significant challenge for any single centre to scan a large sample. Multi-centre studies provide a means of addressing this issue with the pooling of data from different sites to produce relatively large samples.

We adopted this approach in the present study. Our aim was to combine magnetic resonance imaging (MRI) data from multiple centres and measure cortical thickness in large samples of UHR subjects and healthy controls. We also sought to compare cortical thickness in UHR subjects who subsequently did or did not developed psychosis. Whole-brain MRIs were acquired from individuals at UHR for psychosis and healthy controls at four psychiatric research centres in London, Basel, Munich and Melbourne. At each site, subjects were scanned at first clinical presentation, and followed up clinically or in the context of research projects for at least 2 years, so that they could be subcategorized according to psychosis outcome. The MRI data from each site were combined to form a large UHR sample, which was subdivided into subjects who later had developed psychosis and subjects who had not. The thickness of the cerebral cortex was assessed using a voxel-based cortical thickness (VBCT) approach that generates maps from MRI data in which each voxel in the GM is assigned a thickness value and regionally specific differences are compared on a voxel-by-voxel basis (Hutton et al. 2008, 2009). This method differs from others reported in the literature, as it does not require the construction of a threedimensional model for extracting cortical thickness values. For instance, surface-based techniques involve 
the generation of surface models that are driven by image information and surface geometry to fit the GM and white matter (WM) surfaces of the image (Fischl \& Dale, 2000; Hutton et al. 2008). Cortical thickness is subsequently defined at surface points and is computed based on the measure of the distance between them. Another approach involves extracting only the surface between the GM and the WM and then mapping towards the surface the thickness values that are derived by calculating the distance between voxels in the cortex and the surface (Lerch \& Evans, 2005; Hutton et al. 2008). In contrast, using the VBCT technique, GM and WM boundaries are defined on the basis of whole voxel information (Jones et al. 2000; Hutton et al. 2008) and cortical thickness is calculated at every volumetric point within the cortex and based on the length of the trajectory from one boundary to another (Hutton et al. 2008).

Our first prediction, based on data from previous studies (Narr et al. 2005a; Fornito et al. 2008a,b; Schultz et al. 2010b; Jung et al. 2011; Ziermans et al. 2012), was that the UHR group as a whole would show differences in cortical thickness relative to controls in areas that have previously been identified in studies of cortical thickness in UHR subjects and patients with first-episode psychosis: the frontal, anterior cingulate, parahippocampal, temporal, parietal cortices and the precuneus. Our final prediction was that within the UHR sample, subjects who developed psychosis subsequent to scanning would show more pronounced cortical thickness abnormalities in these regions (Narr et al. 2005a; Fornito et al. 2008a,b; Schultz et al. 2010b; Jung et al. 2011; Ziermans et al. 2012) than those who did not.

\section{Method}

\section{Sample}

All the UHR subjects were recruited from specialized clinical services for this group in London [Outreach and Support in South London (OASIS)], Basel [Clinic for Early Detection of Psychosis (FEPSY) at the Psychiatric Outpatient Department, University Hospital], Melbourne [Personal Assessment and Crisis Evaluation (PACE) Clinic] and Munich [Early Detection and Intervention Centre for Mental Crisis (FETZ), Department of Psychiatry and Psychotherapy, Ludwig-Maximilians-University]. Criteria used to identify participants at UHR for psychosis were comparable across the different sites as reported in Supplementary Table S2. Specifically, the London and Melbourne sites used as a screening instrument the Comprehensive Assessment for at Risk Mental State (Yung et al. 2005); the Basel site used as a screening assessment the Basel Screening Instrument for Psychosis (Riecher-Rössler et al. 2008); and the Munich site used the Bonn Scale for the Assessment of Basic Symptoms (Gross et al. 1987). In addition they also assessed attenuated psychotic symptoms and brief limited intermittent psychotic symptoms as defined by the PACE criteria (Yung et al. 2005). Data were combined from these four sites. This sample overlaps with a multi-centre sample that was previously used to investigate GM volume in UHR subjects (Mechelli et al. 2011), and includes subjects that participated in previous single-centre studies of GM volume in this population. In total there were MRI data from 167 UHR subjects. MRI data acquired from healthy volunteers from the same geographical area as the UHR subjects at each site were combined to form a control dataset. Healthy controls were excluded if there was a past or present personal or familiar history of neurological and/or psychiatric conditions. The total control sample comprised 150 subjects, and was comparable with the total UHR sample with respect to gender, age and ethnicity (Table 1).

Subsequent to MRI scanning, the UHR subjects were followed up and assessed regularly for at least 2 years at all four sites. UHR subjects who developed a first episode of psychosis during this period were identified using standardized transition criteria (McGorry et al. 2003; Yung et al. 2004). Each site used this information to subdivide their UHR sample into a group that had made a transition to psychosis (UHR-T), and a group that had not (UHR-NT). In the following 30.6 (S.D. $=10.4)$ months, $50(30 \%)$ of the UHR individuals developed psychosis (UHR-T) and 117 did not (UHR-NT). Transition to psychosis during the follow-up period was established according to Diagnostic and Statistical Manual of Mental Disorders, fourth edition (DSM-IV) criteria based on clinical consensus between at least two experienced psychiatrists. Most of the UHR group (147/167, 84\%) had never taken antipsychotics at time of scanning; $20(12 \%)$ had been exposed to antipsychotics; the mean antipsychotic medication exposure time was 7.5 (S.D. $=11.1)$ weeks. UHR participants that were receiving medication were scanned at the London or Basel sites. Inclusion and exclusion criteria for the two experimental groups, clinical characteristics and sociodemographic of the UHR subjects are reported in the Supplementary material (Supplementary Tables S1-3, S5-8).

\section{Image acquisition}

At all four sites, volumetric MR images were acquired using scanner field strengths of $1.5 \mathrm{~T}$ and a $\mathrm{T}_{1}$-weighted protocol. Two sites used General Electric 
Table 1. Sociodemographic data of the study samples

\begin{tabular}{|c|c|c|c|c|}
\hline Characteristics & $\begin{array}{l}\text { Healthy controls } \\
(n=150)\end{array}$ & $\begin{array}{l}\text { UHR-NT } \\
(n=117)\end{array}$ & $\begin{array}{l}\text { UHR-T } \\
(n=50)\end{array}$ & Significance \\
\hline Mean age, years (s.D.) & $23.4(4.3)$ & $23.3(5.3)$ & $22.9(4.6)$ & $F_{2,314}=0.388, \mathrm{df}=2, p=0.6979$ \\
\hline Gender, $n$ & & & & $\chi^{2}=4.188, \mathrm{df}=2, p=0.123$ \\
\hline Female & 51 & 49 & 13 & \\
\hline Male & 99 & 68 & 37 & \\
\hline \multicolumn{5}{|l|}{ Ethnicity, $n$} \\
\hline Caucasian & 128 & 96 & 43 & $\chi^{2}=8.320, \mathrm{df}=6, p=0.216$ \\
\hline Black & 7 & 5 & 1 & \\
\hline Asian & 9 & 5 & 0 & \\
\hline Mixed & 6 & 11 & 6 & \\
\hline \multicolumn{5}{|l|}{ Handedness, $n$} \\
\hline Right & 132 & 111 & 45 & $\chi^{2}=4.164, \mathrm{df}=4, p=0.384$ \\
\hline Left & 12 & 4 & 4 & \\
\hline Ambidextrous & 6 & 2 & 1 & \\
\hline Medication, $n$ & $\mathrm{~N} / \mathrm{A}$ & 21 & 5 & $\chi^{2}=1.684, \mathrm{df}=1, p=0.194$ \\
\hline
\end{tabular}

UHR-NT, Ultra-high risk without disease transition; UHR-T, UHR with disease transition; S.D., standard deviation; df, degrees of freedom; N/A, not applicable.

scanners (i.e. London and Melbourne) and two used Siemens scanners (i.e. Basel and Munich). The details of the image acquisition sequence are reported in Supplementary Table S4.

\section{Data analysis}

\section{Sociodemographics}

Sociodemographic differences between groups were examined using one-way analysis of variance for parametric data, and by $\chi^{2}$ test for non-parametric data, as implemented in SPSS 19.0 for Windows (IBM, USA) (Table 1, Supplementary Tables S5-8).

\section{Pre-processing}

Pre-processing for the analysis of cortical thickness was carried out using the procedure described by Hutton et al. $(2008,2009)$. In brief, all the images were visually checked and re-sampled to a voxel size of $1 \mathrm{~mm}^{3}$ using trilinear interpolation. Using the unified segmentation procedure implemented in SPM8 (http://www.fil.ion. ucl.ac.uk/spm), the images were segmented into GM, WM and cerebrospinal fluid (CSF) (Ashburner, 2007). For each subject, this resulted in a set of three images in the same space as the original $T_{1}$-weighted image, in which each voxel was assigned a probability of being GM, WM and CSF, respectively. A VBCT map was created for each subject using the GM, WM and CSF segments created in the previous step (Hutton et al. 2008). This method is implemented as a toolbox for SPM (Hutton et al. 2008, 2009). In brief, it uses the input tissue probability maps and a transformed labelled brain atlas (http://www.fil.ion.ucl.ac. uk/spm/ext/\#IBASPM). Starting from the initial estimate of the GM/WM boundary, layers of one voxel in thickness are successively added to surround the WM allowing voxels to be identified where the GM from different sides of a sulcus was in contact. Once all GM voxels have been processed in this way, Laplace's equation is solved for all voxels between the final GM/WM and GM/CSF boundaries resulting in a scalar field that makes a smooth transition from one boundary to the other. The gradient of this field at each point forms a unique trajectory connecting the two boundaries, and the thickness at each point is calculated by integrating along these trajectories. The resulting VBCT maps are created in the space of the original input images which contain cortical thickness values within voxels identified as cortical GM and zeros outside the cortex. DARTEL (Ashburner, 2007), an algorithm for diffeomorphic image registration which is implemented as a toolbox for SPM8, was used to warp the VBCT maps into a new group-specific reference space representing an average of all the subjects. This procedure uses the GM and WM segments estimated from the original $\mathrm{T}_{1}$-weighted images to calculate a group-specific template and the deformation fields required to warp data from each subject to the new template. Each VBCT map was warped to the new template using the corresponding subject-specific deformation field and was re-sampled to an isotropic voxel size of $1.5 \mathrm{~mm}^{3}$ using trilinear interpolation. The warped VBCT maps were scaled by the Jacobian 
determinant of the deformations to account for stretching and compression and subsequently smoothed with a $6 \mathrm{~mm}$ Gaussian kernel then divided by a binary mask of each original VBCT map which had been identically warped, scaled and smoothed (see Supplementary Fig. S1). A Gaussian kernel of $6 \mathrm{~mm}$ was chosen because, when investigating cortical thickness, it is important to keep smoothing to a minimum so that any abrupt changes in thickness that may occur at the boundaries between cortical areas are not obscured (Hutton et al. 2008). This procedure results in smoothed warped VBCT maps for which the Gaussian smoothing kernel applied in the warped space has been projected into the native space of the subject, and the cortical thickness values are preserved over a region the size of the smoothing kernel.

\section{Statistical analysis}

Statistical analysis was performed using SPM8 software (Wellcome Trust Centre for Neuroimaging, UK) on the smoothed warped VBCT maps. An analysis of variance was used to compare cortical thickness in UHR-T, UHR-NT and healthy control subjects. Scanner site was modelled as an additional factor, resulting in a total of 12 experimental groups. Including scanner site as a factor in the statistical analysis allowed us to model scanner-related variance in the data, which had the effect of reducing error variance and increasing statistical sensitivity. We also modelled age, gender, ethnicity and handedness as covariates of no interest to minimize any confounding effect of these variables on the findings. An ROI approach was used to examine between-group differences in areas where abnormalities in cortical thickness have been identified in MRI studies of individuals at UHR or with a first episode of psychosis, excluding studies that included data from subjects who participated in the present investigation. These comprised the parahippocampal gyrus, inferior frontal gyrus, anterior cingulate, superior temporal gyrus, inferior parietal gyrus and the precuneus (Narr et al. 2005a; Schultz et al. 2010b; Jung et al. 2011; Ziermans et al. 2012). Using WFU PickAtlas (http://www.nitrc.org/ projects/wfu_pickatlas/) we created a mask that included the six chosen ROIs and comprised a total of 11700 voxels. Within this mask, statistical inferences were made using a statistical threshold of $p<0.05$ after familywise error (FWE) correction for multiple comparisons as calculated in SPM8. Trends that did not survive correction for multiple comparisons ( $p<0.001$ uncorrected) are reported but not discussed. For completeness we also performed a whole-brain analysis using a statistical threshold of $p<0.05$ after FWE correction for multiple comparisons.

\section{Results}

\section{Sociodemographics}

No statistically significant differences were noted among the UHR-T, UHR-NT and control groups in age, gender, ethnicity and handedness (Table 1).

\section{Differences in cortical thickness between UHR subjects and healthy controls}

Within the ROIs, the cortex was thinner in the UHR group than in controls in the right parahippocampal gyrus $[p<0.05$ FWE corrected; $z$ score $=4.06$; Montreal Neurological Institute (MNI) coordinates $x=25, y=-4$, $\mathrm{z}=-13$; see Fig. 1]. There was also a trend $(p<0.001$ uncorrected) for a thinner cortex in the UHR group compared with controls in the inferior part of the left parahippocampal gyrus $(z$ score $=3.42$; MNI coordinates $x=-20, y=-7, z=-30)$. Plotting of the cortical thickness values revealed that the reduction in right parahippocampal cortical thickness was evident in the data from each of the four sites (Fig. 1). In contrast, there were no areas in which UHR individuals had a thicker cortex than healthy controls. The whole-brain analysis did not identify significant differences in cortical thickness between the UHR group and healthy controls at $p<0.05$ (FWE corrected).

\section{Differences in cortical thickness between the UHR-T and UHR-NT groups}

No differences were observed for the comparison between the UHR-T and UHR-NT groups at $p<0.05$ after FWE correction. At a less conservative statistical threshold ( $p<0.001$ uncorrected), there was a trend for cortical thinning in the UHR-T group in the orbital part of the left inferior frontal gyrus $(z$ score $=3.32$; MNI coordinates $x=-33, y=32, z=-15)$. The wholebrain analysis revealed no significant differences between the UHR-T and UHR-NT groups at $p<0.05$ (FWE corrected).

\section{Discussion}

Previous neuroimaging studies have reported cortical thinning in schizophrenia, as well as in first-episode psychosis and in subjects at UHR for psychosis (Narr et al. 2005a; Fornito et al. 2008a,b; Schultz et al. 2010b; Jung et al. 2011; Ziermans et al. 2012). However, the results of these studies have not always been consistent; this may reflect the recruitment of relatively small samples, the use of different study designs, and the investigation of samples that were heterogeneous with respect to age, duration of illness and exposure to treatment. In the present study, we sought to reduce the impact of these potential methodological 


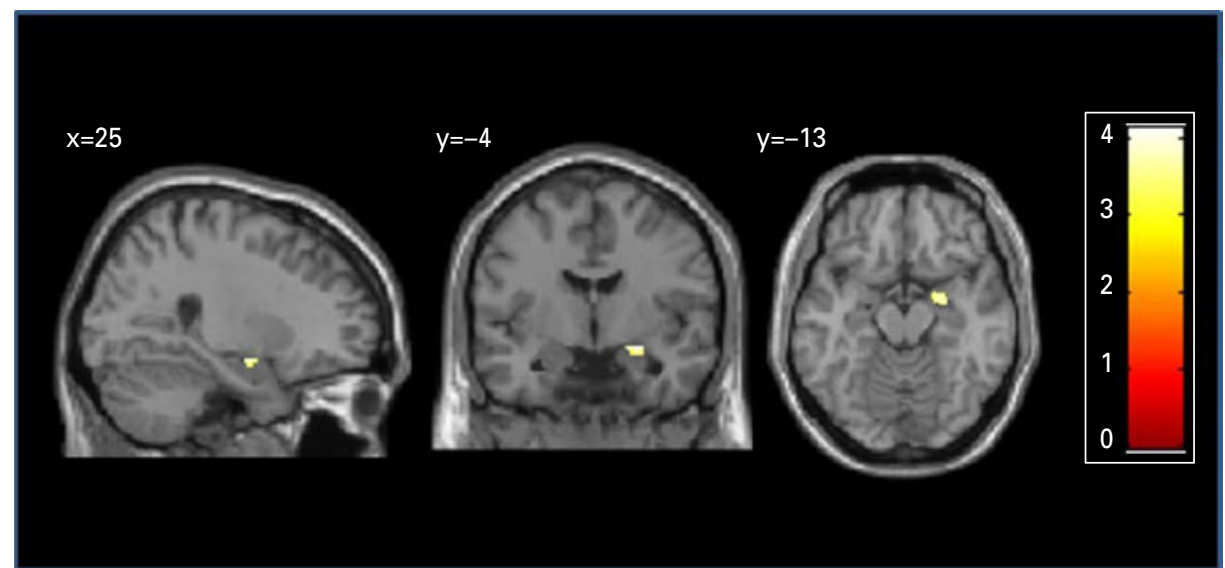

5

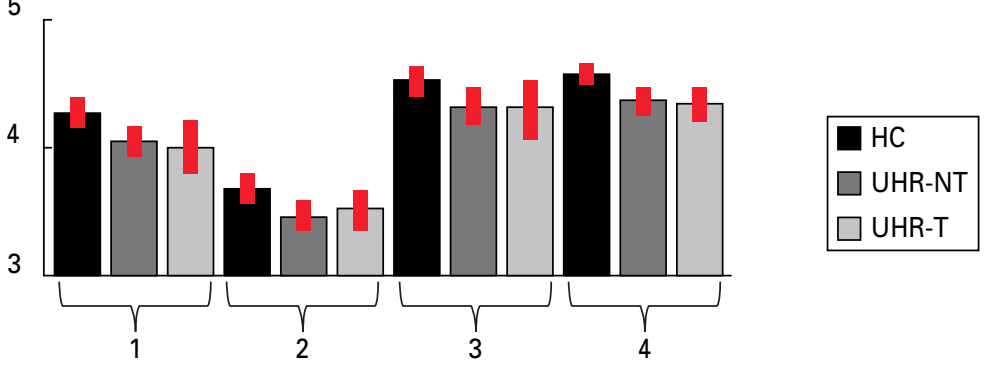

Fig. 1. Cortical thickness differences between the ultra-high risk (UHR) and healthy control (HC) groups: right parahippocampal region where the total UHR sample showed cortical thinning relative to HCs $(p<0.05$ after familywise error correction). For visualization purposes, effects are displayed at $p<0.001$ uncorrected. The plot shows cortical thickness values for the HC group and the two UHR subgroups (UHR-T, transition to psychosis; UHR-NT, no transition to psychosis) at each site $(x$ axis: $1=$ London; $2=$ Basel; $3=$ Melbourne; $4=$ Munich); values on the $y$ axis refer to millimetres. Error bars represent standard deviations.

pitfalls by assessing cortical thickness in a relatively large sample of individuals at UHR for psychosis, all of whom were scanned at a similar stage of illness, when they first presented with clinical high-risk symptoms. Most of the sample had not been treated before. Our first hypothesis was that the UHR group as a whole would show differences in regional cortical thickness relative to controls, and that these would be most evident in areas that have previously been identified as sites of cortical thickness or volume abnormalities in studies of UHR and first-episode subjects (i.e. the temporal, frontal, parietal, anterior cingulate, parahippocampal cortices and the precuneus). This hypothesis was in part confirmed, in that we found that the right parahippocampal cortex was thinner in the UHR group than in controls. This finding is consistent with those of a recent investigation which also found reductions in thickness in other cortical areas (Jung et al. 2011). Our result is also in line with evidence that the density of the parahippocampal gyrus is altered in UHR and familial high-risk subjects (Job et al. 2003). Furthermore, in first-episode schizophrenia, altered right parahippocampal-lingual cortical folding and reduced cortical thickness have been found (Schultz et al. 2010a). Finally, this region has also been identified as a site of functional (Allen et al. 2011) alterations in UHR subjects and is one of the most robust site of volume reduction (Seidman et al. 2003) and neuropathological abnormalities (Shenton et al. 2001) in schizophrenia.

In our previous volumetric study in an UHR sample that overlapped with that in the present study, we found differences between the UHR sample and controls in the ventral prefrontal cortex and ACC, but not in the parahippocampal cortex (Mechelli et al. 2011). However, in that study the subgroup of UHR subjects that subsequently developed psychosis had smaller left parahippocampal volumes than the UHR subjects who did not make a transition to psychosis. This volumetric finding was in a similar part of the parahippocampal gyrus to the site of the difference in cortical thickness between all UHR subjects and controls in the present study, although in the opposite hemisphere. Differences in the location of alterations in cortical thickness and cortical volume may reflect the fact that abnormalities in these two measures are 
not necessarily manifestations of the same underlying neuroanatomical changes. This would be consistent with recent evidence that cortical thickness and surface area are genetically and phenotypically independent and that the local cortical volume is more closely related to surface area than to cortical thickness (Winkler et al. 2010). Therefore differences between the results of our present study and our previous VBM study may be partially explained by the fact that the cortical thickness method does not take surface information into account. The volumetric reduction of the left parahippocampal gyrus reported in our VBM study (Mechelli et al. 2011) may denote a marker of progression consistent with the results of earlier investigations (Pantelis et al. 2003; Job et al. 2005), whereas the thinning of the right parahippocampal gyrus may represent either a vulnerability marker (evident not only in the UHR stage but also in the earlier asymptomatic stage) or a specific marker of the UHR stage (not evident in the earlier asymptomatic stage). These two alternative options could be investigated by acquiring longitudinal neuroimaging data from individuals at high genetic risk.

Our second hypothesis was that UHR subjects who went on to develop psychosis would already at baseline show more pronounced cortical thickness abnormalities than UHR subjects who did not. We did not find any differences that survived correction for multiple comparisons. Two previous studies have investigated cortical thickness in UHR subjects in relation to clinical outcome. One study, which restricted its analysis to the ACC, reported cortical thinning in this region in the UHR-T group compared with the UHR-NT group (Fornito et al. 2008b). We included the ACC as one of our ROIs but did not replicate this finding, although this may be in part explained by methodological differences in thickness measurements and in ROIs investigated. The second study used MRI to scan UHR subjects at two time points and compared longitudinal changes in cortical thickness in UHR individuals who did and did not make a transition to psychosis. The UHR-T group showed longitudinal reductions in several regions including anterior cingulate, precuneus and temporo-parietal-occipital areas compared with controls, whereas the UHR-NT group did not (Ziermans et al. 2012). In contrast, no differences in cortical thickness were reported between UHR-T and UHR-NT subjects at baseline. The inconsistency between previous studies and our investigation could be in part explained by the use of different techniques to measure cortical thickness. In particular, the studies by Fornito and colleagues and Ziermans and colleagues used two different surfacebased methods (Fischl \& Dale, 2000; Kim et al. 2005; Fornito et al. 2008b; Ziermans et al. 2012) while in the present investigation, a voxel-based method was used to derived cortical thickness values (Hutton et al. 2008). Another study reported no baseline differences in cortical thickness between UHR and firstepisode psychosis and healthy controls, although the analysis of cortical asymmetry revealed significant group differences (Haller et al. 2009). The lack of significant differences between UHR-T and UHR-NT subjects may seem surprising, given that the present sample was relatively large, and abnormalities in functional and in other structural measures between these subgroups have previously been identified (Pantelis et al. 2003; Borgwardt et al. 2008, Mechelli et al. 2011; Allen et al. 2012). One possible explanation for the absence of significant differences in the present study is that there was some heterogeneity within our UHR sample. Individuals can meet the UHR inclusion criteria through different patterns of clinical and cognitive symptoms, and it is unknown if these reflect distinct pathophysiological processes. Unfortunately, a stratification of the statistical analysis by type of UHR inclusion criteria was not possible in the present investigation, as incorporating this additional factor would require much larger single-centre sample sizes. The sample may also have been clinically heterogeneous with respect to the co-morbid anxiety, depression and substance abuse that are often present in UHR subjects (Svirskis et al. 2005).

Another possibility is that some subjects who met transition criteria subsequently have returned to lower levels of psychotic symptoms and higher levels of functioning, while some who did not meet criteria for transition actually have deteriorated in functioning over time. This latter group may be more likely to develop schizophrenia than the former group (Yung et al. 2010). Thus, the non-transitioned but low functioning group may show brain imaging changes consistent with the ones observed in schizophrenia, but the high functioning transitioned cases may not.

The age range of our UHR individuals varied considerably across the four sites (age range 15-37 years). Previous work indicates that the pattern of neuroanatomical findings in schizophrenia with a relatively early onset (e.g. before the age of 18 years) differs from that in schizophrenia with onset in adulthood and that the longitudinal trajectory of brain abnormalities varies with the age of onset (Gogtay et al. 2011). Although we modelled age as a covariate of no interest in the statistical analysis, the fact that the UHR sample comprised individuals at different stages of brain development might have reduced the likelihood of detecting reliable differences.

A further potential contributory factor is that the MRI data we studied were collected on different scanners, using different acquisition sequences. However, 
we are confident that our results were not significantly affected by the use of different scanners for several reasons. First, only MRI data collected with $\mathrm{T}_{1}$-weighted sequences were used and scanner site was modelled as an independent factor in the statistical analysis. Second, a comparable proportion of control, UHR-NT and UHR-T subjects were scanned at each scanning site. Third, plotting of GM values suggested that the differences between UHR and healthy control groups were evident also within each site and therefore cannot be explained by inter-scanner differences (Fig. 1). Finally, the present approach to the integration of multi-scanner data has been employed successfully in previous studies that also combined different scanners and acquisition sequences (Stonnington et al. 2008; Segall et al. 2009; Suckling et al. 2010). Nevertheless, we cannot completely exclude an effect of scanner on the findings, and ideally multicentre studies should employ the same acquisition sequence, and calibrate data across scanners using phantoms and common subjects to minimize the risk of scanner-related effects (Jack et al. 2008).

The whole-brain analysis did not identify significant differences in cortical thickness between the UHR group and healthy controls or between UHR-T and UHR-NT subjects at $p<0.05$ (FWE corrected). This could be in part explained by the heterogeneity of the UHR sample (e.g. symptoms, level of functioning, age), or by the use of different scanners and sequences. Nevertheless, when restricting the analysis to ROIs, the method employed was able to detect a thickness reduction in the right parahippocampal gyrus in the UHR group compared with the control group at a statistical threshold of $p<0.05$ corrected (FWE). The reduction was evident across all the different scanning sites. Using the whole-brain approach did not enable us to detect differences at a corrected level; this might indicate that group differences in the UHR population are distributed and diluted across the brain cortex.

In conclusion, we observed right parahippocampal thinning in subjects at UHR for psychosis, suggesting that thickness reduction in this region is related to the UHR symptomatology rather than the onset of psychosis. This cortical alteration could represent a marker of vulnerability to psychosis or, alternatively, a specific and distinctive marker of the UHR stage. No reliable differences in cortical thickness were found between subjects who did and did not go on to develop psychosis. Future multi-centre work in this area would benefit from the subcharacterization of UHR individuals with different clinical and cognitive profiles, the recruitment of participants at the same stage of neurodevelopment and the use of a standardized image acquisition sequence.

\section{Supplementary material}

For supplementary material accompanying this paper visit http://dx.doi.org/10.1017/S0033291713000998.

\section{Acknowledgements}

This work was supported by the National Alliance for Research on Schizophrenia and Depression Independent Investigator Award to A.M.; the National Health and Medical Research Council of Australia (grant no. 970391, 981112, 145737, 145627, 350241, 566529); the National Health and Medical Research Council Clinical Career Developmental Award (grant no. 359223 to S.W.); the National Alliance for Research on Schizophrenia and Depression Young Investigator Award to S.W.; the National Health and Medical Research Council Senior Principal Research Fellowship (grant no. 628386 to C.P.); the National Alliance for Research on Schizophrenia and Depression Distinguished Investigator Award to C.P.; the National Health and Medical Research Council Senior Research Fellowship (grant no. 566593 to A.Y.) and the Wellcome Trust (grant no. 091593/Z/10/Z to C.H.).

\section{Declaration of Interest}

None.

\section{References}

Allen P, Luigjes J, Howes OD, Egerton A, Hirao K, Valli I, Kambeitz J, Fusar-Poli P, Broome M, McGuire P (2012). Transition to psychosis associated with prefrontal and subcortical dysfunction in ultra high-risk individuals. Schizophrenia Bulletin 38, 1268-1276.

Allen P, Seal ML, Valli I, Fusar-Poli P, Perlini C, Day F, Wood SJ, Williams SC, McGuire PK (2011). Altered prefrontal and hippocampal function during verbal encoding and recognition in people with prodromal symptoms of psychosis. Schizophrenia Bulletin 37, 746-756.

Ashburner J (2007). A fast diffeomorphic image registration algorithm. Neuroimage 38, 95-113.

Borgwardt SJ, McGuire PK, Aston J, Gschwandtner U, Pflüger MO, Stieglitz RD, Radue EW, Riecher-Rössler A (2008). Reductions in frontal, temporal and parietal volume associated with the onset of psychosis. Schizophrenia Research 106, 108-114.

Buehlmann E, Berger GE, Aston J, Gschwandtner U, Pflueger MO, Borgwardt SJ, Radue EW, Riecher-Rössler A (2010). Hippocampus abnormalities in at risk mental states for psychosis? A cross-sectional high resolution region of interest magnetic resonance imaging study. Journal of Psychiatric Research 44, 447-453.

Büschlen J, Berger GE, Borgwardt SJ, Aston J, Gschwandtner U, Pflueger MO, Kuster P, Radü EW, Stieglitz RD, Riecher-Rössler A (2011). Pituitary volume 
increase during emerging psychosis. Schizophrenia Research 125, 41-48.

Fischl B, Dale AM (2000). Measuring the thickness of the human cerebral cortex from magnetic resonance images. Proceedings of the National Academy of Sciences USA 97, 11050-11055.

Fornito A, Yucel M, Wood SJ, Adamson C, Velakoulis D, Saling MM, McGorry PD, Pantelis C (2008a).

Surface-based morphometry of the anterior cingulate cortex in first episode schizophrenia. Human Brain Mapping 29, 478-489.

Fornito A, Yung AR, Wood SJ, Phillips LJ, Nelson B, Cotton S, Velakoulis D, McGorry PD, Pantelis C, Yucel M (2008b). Anatomic abnormalities of the anterior cingulate cortex before psychosis onset: an MRI study of ultra-high-risk individuals. Biological Psychiatry 64, 758-765.

Fusar-Poli P, Bonoldi I, Yung AR, Borgwardt S, Kempton MJ, Valmaggia L, Barale F, Caverzasi E, McGuire P (2012). Predicting psychosis: meta-analysis of transition outcomes in individuals at high clinical risk. Archives of General Psychiatry 69, 220-229.

Gogtay N,Vyas NS,Testa R,Wood SJ,Pantelis C (2011). Age of onset of schizophrenia: perspectives from structural neuroimaging studies. Schizophrenia Bulletin 37, 504-513.

Gross G, Huber G, Klosterkotter J (1987). Bonn Scale for the Assessment of Basic Symptoms - BSABS. Springer: Berlin.

Haller S, Borgwardt SJ, Schindler C, Aston J, Radue EW, Riecher-Rössler A (2009). Can cortical thickness asymmetry analysis contribute to detection of at-risk mental state and first-episode psychosis? A pilot study. Radiology 250, 212-221.

Hutton C, De Vita E, Ashburner J, Deichmann R, Turner R (2008). Voxel-based cortical thickness measurements in MRI. Neuroimage 40, 1701-1710.

Hutton C, Draganski B, Ashburner J, Weiskopf N (2009). A comparison between voxel-based cortical thickness and voxel-based morphometry in normal aging. Neuroimage 48, 371-380.

Jack CR Jr, Bernstein MA, Fox NC, Thompson P, Alexander G, Harvey D, Borowski B, Britson PJ, Whitwell JL, Ward C, Dale AM, Felmlee JP, Gunter JL, Hill DL, Killiany R, Schuff N, Fox-Bosetti S, Lin C, Studholme C, DeCarli CS, Krueger G, Ward HA, Metzger GJ, Scott KT, Mallozzi R, Blezek D, Levy J, Debbins JP, Fleisher AS, Albert M, Green R, Bartzokis G, Glover G, Mugler J, Weiner MW (2008). The Alzheimer's Disease Neuroimaging Initiative (ADNI): MRI methods. Journal of Magnetic Resonance Imaging 27, 685-691.

Job DE, Whalley HC, Johnstone EC, Lawrie SM (2005). Grey matter changes over time in high risk subjects developing schizophrenia. Neuroimage 25, 1023-1030.

Job DE, Whalley HC, McConnell S, Glabus M, Johnstone EC, Lawrie SM (2003). Voxel-based morphometry of grey matter densities in subjects at high risk of schizophrenia. Schizophrenia Research 64, 1-13.

Jones SE, Buchbinder BR, Aharon I (2000).

Three-dimensional mapping of cortical thickness using Laplace's equation. Human Brain Mapping 11, 12-32.
Jung WH, Kim JS, Jang JH, Choi JS, Jung MH, Park JY, Han JY, Choi CH, Kang DH, Chung CK, Kwon JS (2011). Cortical thickness reduction in individuals at ultra-high-risk for psychosis. Schizophrenia Bulletin 37, 839-849.

Kim JS, Singh V, Lee JK, Lerch J, Ad-Dab'bagh Y, MacDonald D, Lee JM, Kim SI, Evans AC (2005). Automated 3-D extraction and evaluation of the inner and outer cortical surfaces using a Laplacian map and partial volume effect classification. Neuroimage 27, 210-221.

Koutsouleris N, Meisenzahl EM, Davatzikos C, Bottlender R, Frodl T, Scheuerecker J, Schmitt G, Zetzsche T, Decker P, Reiser M, Moller HJ, Gaser C (2009). Use of neuroanatomical pattern classification to identify subjects in at-risk mental states of psychosis and predict disease transition. Archives of General Psychiatry 66, 700-712.

Lerch JP, Evans AC (2005). Cortical thickness analysis examined through power analysis and a population simulation. Neuroimage 24, 163-173.

McGorry PD, Nelson B, Amminger GP, Bechdolf A, Francey SM, Berger G, Riecher-Rössler A, Klosterkotter J, Ruhrmann S, Schultze-Lutter F, Nordentoft M, Hickie I, McGuire P, Berk M, Chen EY, Keshavan MS, Yung AR (2009). Intervention in individuals at ultra-high risk for psychosis: a review and future directions. Journal of Clinical Psychiatry 70, 1206-1212.

McGorry PD, Yung AR, Phillips LJ (2003). The "close-in" or ultra high-risk model: a safe and effective strategy for research and clinical intervention in prepsychotic mental disorder. Schizophrenia Bulletin 29, 771-790.

Mechelli A, Riecher-Rössler A, Meisenzahl EM, Tognin S, Wood SJ, Borgwardt SJ, Koutsouleris N, Yung AR, Stone JM, Phillips LJ, McGorry PD, Valli I, Velakoulis D, Woolley J, Pantelis C, McGuire P (2011).

Neuroanatomical abnormalities that predate the onset of psychosis: a multicenter study. Archives of General Psychiatry 68, 489-495.

Meisenzahl EM, Koutsouleris N, Gaser C, Bottlender R, Schmitt GJ, McGuire P, Decker P, Burgermeister B, Born C, Reiser M, Möller HJ (2008). Structural brain alterations in subjects at high-risk of psychosis: a voxel-based morphometric study. Schizophrenia Research 102, 150-162.

Narr KL, Bilder RM, Toga AW, Woods RP, Rex DE, Szeszko PR, Robinson D, Sevy S, Gunduz-Bruce H, Wang Y-P, DeLuca H, Thompson PM (2005a). Mapping cortical thickness and gray matter concentration in first episode schizophrenia. Cerebral Cortex 15, 708-719.

Narr KL, Toga AW, Szeszko P, Thompson PM, Woods RP, Robinson D, Sevy S, Wang Y, Schrock K, Bilder RM (2005b). Cortical thinning in cingulate and occipital cortices in first episode schizophrenia. Biological Psychiatry 58, 32-40.

Pantelis C, Velakoulis D, McGorry PD, Wood SJ, Suckling J, Phillips LJ, Yung AR, Bullmore ET, Brewer W, Soulsby B, Desmond P, McGuire PK (2003).

Neuroanatomical abnormalities before and after onset of psychosis: a cross-sectional and longitudinal MRI comparison. Lancet 361, 281-288. 
Phillips LJ, Velakoulis D, Pantelis C, Wood S, Yuen HP, Yung AR, Desmond P, Brewer W, McGorry PD (2002). Non-reduction in hippocampal volume is associated with higher risk of psychosis. Schizophrenia Research 58, 145-158.

Riecher-Rössler A, Aston J, Ventura J, Merlo M, Borgwardt S, Gschwandtner U, Stieglitz RD (2008). The Basel Screening Instrument for Psychosis (BSIP): development, structure, reliability and validity [article in German]. Fortschritte der Neurologie-Psychiatrie 76, 207-216. Röthlisberger M, Riecher-Rössler A, Aston J, Fusar-Poli P, Radü EW, Borgwardt S (2012). Cingulate volume abnormalities in emerging psychosis. Current Pharmaceutical Design 18, 495-504.

Schultz CC, Koch K, Wagner G, Roebel M, Nenadic I, Gaser C, Schachtzabel C, Reichenbach JR, Sauer H, Schlosser RG (2010a). Increased parahippocampal and lingual gyrification in first-episode schizophrenia. Schizophrenia Research 123, 137-144.

Schultz CC, Koch K, Wagner G, Roebel M, Schachtzabel C, Gaser C, Nenadic I, Reichenbach JR, Sauer H, Schlosser RG (2010b). Reduced cortical thickness in first episode schizophrenia. Schizophrenia Research 116, 204-209.

Segall JM, Turner JA, van Erp TG, White T, Bockholt HJ, Gollub RL, Ho BC, Magnotta V, Jung RE, McCarley RW, Schulz SC, Lauriello J, Clark VP, Voyvodic JT, Diaz MT, Calhoun VD (2009). Voxel-based morphometric multisite collaborative study on schizophrenia. Schizophrenia Bulletin 35, 82-95.

Seidman LJ, Pantelis C, Keshavan MS, Faraone SV, Goldstein JM, Horton NJ, Makris N, Falkai P, Caviness VS, Tsuang MT (2003). A review and new report of medial temporal lobe dysfunction as a vulnerability indicator for schizophrenia: a magnetic resonance imaging morphometric family study of the parahippocampal gyrus. Schizophrenia Bulletin 29, 803-830.

Shenton ME, Dickey CC, Frumin M, McCarley RW (2001). A review of MRI findings in schizophrenia. Schizophrenia Research 49, 1-52.

Stonnington CM, Tan G, Kloppel S, Chu C, Draganski B, Jack CR Jr, Chen K, Ashburner J, Frackowiak RS (2008). Interpreting scan data acquired from multiple scanners: a study with Alzheimer's disease. Neuroimage 39, 1180-1185.

Suckling J, Barnes A, Job D, Brenan D, Lymer K, Dazzan P, Marques TR, MacKay C, McKie S, Williams SR, Williams SC, Lawrie S, Deakin B (2010). Power calculations for multicenter imaging studies controlled by the false discovery rate. Human Brain Mapping 31, 1183-1195.

Svirskis T, Korkeila J, Heinimaa M, Huttunen J, Ilonen T, Ristkari T, McGlashan T, Salokangas RK (2005). Axis-I disorders and vulnerability to psychosis. Schizophrenia Research 75, 439-446.

Takahashi T, Wood SJ, Yung AR, Phillips LJ, Soulsby B, McGorry PD, Tanino R, Zhou SY, Suzuki M,
Velakoulis D, Pantelis C (2009). Insular cortex gray matter changes in individuals at ultra-high-risk of developing psychosis. Schizophrenia Research 111, 94-102.

Takahashi T, Wood SJ, Yung AR, Walterfang M, Phillips LJ, Soulsby B, Kawasaki Y, McGorry PD, Suzuki M, Velakoulis D, Pantelis C (2010). Superior temporal gyrus volume in antipsychotic-naive people at risk of psychosis. British Journal of Psychiatry 196, 206-211.

Velakoulis D, Wood SJ, Wong MT, McGorry PD, Yung A, Phillips L, Smith D, Brewer W, Proffitt T, Desmond P, Pantelis C (2006). Hippocampal and amygdala volumes according to psychosis stage and diagnosis: a magnetic resonance imaging study of chronic schizophrenia, first-episode psychosis, and ultra-high-risk individuals. Archives of General Psychiatry 63, 139-149.

Venkatasubramanian G, Jayakumar PN, Gangadhar BN, Keshavan MS (2008). Automated MRI parcellation study of regional volume and thickness of prefrontal cortex (PFC) in antipsychotic-naive schizophrenia. Acta Psychiatrica Scandinavica 117, 420-431.

Winkler AM, Kochunov P, Blangero J, Almasy L, Zilles K, Fox PT, Duggirala R, Glahn DC (2010). Cortical thickness or grey matter volume? The importance of selecting the phenotype for imaging genetics studies. Neuroimage 53, 1135-1146.

Wood SJ, Yucel M, Velakoulis D, Phillips LJ, Yung AR, Brewer W, McGorry PD, Pantelis C (2005). Hippocampal and anterior cingulate morphology in subjects at ultra-high-risk for psychosis: the role of family history of psychotic illness. Schizophrenia Research 75, 295-301.

Yucel M, Wood SJ, Phillips LJ, Stuart GW, Smith DJ, Yung A, Velakoulis D, McGorry PD, Pantelis C (2003). Morphology of the anterior cingulate cortex in young men at ultra-high risk of developing a psychotic illness. British Journal of Psychiatry 182, 518-524.

Yung AR, Nelson B, Thompson A, Wood SJ (2010). The psychosis threshold in Ultra High Risk (prodromal) research: is it valid? Schizophrenia Research 120, 1-6.

Yung AR, Phillips LJ, Yuen HP, McGorry PD (2004). Risk factors for psychosis in an ultra high-risk group: psychopathology and clinical features. Schizophrenia Research 67, 131-142.

Yung AR, Yuen HP, McGorry PD, Phillips LJ, Kelly D, Dell'Olio M, Francey SM, Cosgrave EM, Killackey E, Stanford C, Godfrey K, Buckby J (2005). Mapping the onset of psychosis: the Comprehensive Assessment of At-Risk Mental States. Australian and New Zealand Journal of Psychiatry 39, 964-971.

Ziermans TB, Schothorst PF, Schnack HG, Koolschijn PC, Kahn RS, van Engeland H, Durston S (2012). Progressive structural brain changes during development of psychosis. Schizophrenia Bulletin 38, 519-530. 